\title{
The molecules of persuasion-how cells talk to each other
}

\author{
Peter Nick
}

Published online: 10 March 2010

(C) Springer-Verlag 2010

The emergence of multicellularity was a key step in evolution, because it allows to assign different functions to individual cells. However, for the individual cell, differentiation represents a risky investment, because it implies that specific so-called hypercellular functions have to be upregulated at the cost of other hypocellular functions that are downregulated and therefore have to be compensated by corresponding hypercellular output from neighbouring cells (Lintilhac 1999). As a consequence, the individual cell cannot survive outside the organismal context. Differentiation therefore requires an intensive communication between individual cells to maintain the balance between hypercellular and hypocellular functions. Already the prokaryotic cyanobacteria have developed such communication to regulate differentiation: cyanobacterial heterocysts express, as a hypercellular function, nitrogenase that overcomes the limitations in bioavailable nitrogen. This nitrogenase dates back to the earliest, anoxic phases of life and is therefore highly sensitive to oxygen. Thus, to safeguard nitrogenase activity, photosynthetic activity has to be excluded from heterocysts. These cells are therefore hypocellular with respect to assimilation. The balance between nitrogen export and assimilate import has to be maintained although the total number of cells grows continuously. This balance is regulated by diffusible peptides that are released by developing heterocysts and that inhibit the commitment for the heterocyst programme in the neighbouring cells (Yoon and Golden 1998).

\section{P. Nick $(\bowtie)$}

Molecular Cell Biology, Botanical Institute,

Karlsruhe Institute of Technology,

Kaiserstr. 2,

76128 Karlsruhe, Germany

e-mail: peter.nick@bio.uka.de
Intercellular communication has accompanied and driven evolution in all eukaryotes. Three review articles in the present issue highlight different facets of this communication.

The primordial form of cell differentiation is the developmental dichotomy that separates a differentiating daughter cell from a non-differentiating stem cell. The differentiating daughter cell will partially lose its developmental potential, whereas the stem cell will maintain the full developmental potential of the mother cell. In plants, this fundamental event takes place in the meristems and has been shown to be regulated by the peptide CLAVATA3 that is a ligand of the WUSCHEL receptor. However, there exists a whole family of related peptides that seem to be mostly plant specific. The contribution by Fiers and Wang in this issue gives a survey on these peptides, their potential receptors and functions.

Communication does not only rely on a common language, it also requires appropriate channels of communication. Even in the very simple heterocyst systems, the inhibitory peptide signal is channelled parallel to the file axis by extracellular barriers. In multicellular organisms, cytoplasmic bridges have evolved as specific and regulated channels of communication. The review by Verchot-Lubicz and Goldstein in this issue compares the function of these bridges across aquatic and land plants, but also developing oocytes of Caenorhabditis elegans and explores conserved aspects of intercellular communication across cytoplasmic bridges such as the role of molecular motors, cyclosis, ion gradients and membrane dynamics.

Communication has always been sensitive to usurpationan ancient and common strategy of parasitic organisms. A striking example is reviewed in the contribution by Baumeister et al. in this issue. They explain how the apicomplexan malaria parasite overwhelms mammalian 
erythrocytes. Interestingly, the erythrocyte is a strongly reduced cell without an own genetic programme, which means that the parasite has to reprogramme the host cell such that it acquires nutrients from the bloodstream, which is nothing less than compensating the host-cell functions that had been lost during its differentiation.

\section{References}

Lintilhac PM (1999) Towards a theory of cellularity speculations on the nature of the living cell. Bioscience 49:60-68

Yoon HS, Golden JW (1998) Heterocyst pattern formation controlled by a diffusible peptide. Science 282:935-938 